Pacific Journal of Mathematic 


\section{REARRANGING FOURIER TRANSFORMS ON GROUPS}

\section{Chung LiN}

Let $G$ denote an infinite locally compact abelian group and $X$ its character group. Let $\theta$ be a suitable Haar measure on $X$, and $1<p<2$. For a $\theta$-measurable function $\phi$ on $X$, we define $\theta_{\phi}(t)=(\{\chi \in X:|\phi(\chi)|>t\})$ and $\phi^{*}(x)=\inf \left\{t>0: \theta_{\phi}(t) \leqq\right.$ $x$ for $x>0, \phi^{*}$ is called the nonincreasing rearrangement of $\phi$. Note that even though $\phi$ is defined on $X$, the domain of $\phi^{*}$ is $(0, \infty)$. A nonnegative function $g$ defined on $(0, \infty)$ is called admissible if $g$ is nonincreasing and $\lim _{x \rightarrow \infty} g(x)=0$. Theorems:

1. Let $G$ be nondiscrete with a compact open subgroup and $g$ admissible. Then $\left.g\right|_{N}=\left.\hat{f} *\right|_{N}$, where $N$ is the set of positive integers, for some $f \in L^{p}(G)$ if $\sum_{k=1}^{\infty} g(k)^{p} k^{p-2}<\infty$.

2. Let $G$ be nondiscrete with no compact open subgroup and $g$ admissible. Then $g=\hat{f}^{*} m$ a.e. for some $f \in L^{p}(G)$ if $\int_{0}^{\infty} g(x)^{p} x^{p-2} d x<\infty$

3. Let $G$ be an infinite discrete abelian group which contains $Z, Z\left(r^{\infty}\right)$ or $Z(r)^{\aleph_{0}}$ as a subgroup, $g$ admissible. Then $\left.g\right|_{(0,1)}=\left.\hat{f}^{*}\right|_{(0,1)} m$ a.e. for some $f \in L^{p}(G)$ if $\int_{0}^{1} g(x)^{p} x^{p-2} d x<\infty$.

I. Introduction. As usual the Fourier transform $\hat{f}$ of a function $f \in L^{1}(G)$ is defined on $X$ such that $\hat{f}(\chi)=\int_{G} f \chi d \lambda$, where $\lambda$ is a fixed but arbitrary Haar measure on $G$. For $1<p<2, \hat{f} \in L^{p^{\prime}}(G)$ and $p^{\prime}$ is the conjugate exponent of $p$. The set of real numbers, $n$-dimensional Euclidean space, the circle group, the integers, the $r$ adic integers, the countable product of the group of integers modula $r$ and the subgroup of the circle whose elements have order a power of $r$ are denoted by $R, R^{n}, T, Z, \Delta_{r}, \Pi Z(r)$ and $Z\left(r^{\infty}\right)$, respectively. Also $p$ will denote any number such that $1<p<2$. Let $m$ be $1 / \sqrt{2 \pi}$. Lebesque measure on $R$.

Hardy and Littlewood [1], [2] characterized functions on $Z$ such that every rearrangement is the Fourier transform of a function in $L^{p}(T), 2<p<\infty$. They also characterized functions on $Z$ such that some rearrangement is the Fourier transform of afunction in $L^{p}(T)$, $1<p<2$. Hewitt and Ross [4] generalized these results to arbitrary compact infinite abelian groups. We are interested in the case of LCA (locally compact abelian) groups. Here are our results.

THEOREM 1. Let $G$ be nondiscrete with a compact open subgroup, 
and $g$ an admissible function. Then $\left.g\right|_{N}=\left.\hat{f}^{*}\right|_{N}$ for some $f \in L^{p}(G)$ if and only if $\sum_{k=1}^{\infty} g(k)^{p} k^{p-2}<\infty$. Moreover, there exists a constant $A_{p}$ that depends on $p$ only such that

$$
\left(\sum_{k=1}^{\infty} g(k)^{p} k^{p-2}\right)^{1 / p} \leqq A_{p}\|f\|_{p}
$$

for evrey such $f$.

THEOREM 2. Let $G$ be a nondiscrete LCA group with no compact open subgroup and $g$ an admissible function. Then $g=\hat{f}^{*}$ for some $f \in L^{p}(G)$ if and only if $\int_{0}^{\infty} g(x)^{p} x^{p-2} d x<\infty$. Moreover, there exists $A_{p}$ that depends only on $p$ such that

$$
\left(\int_{0}^{\infty} g(x)^{p} x^{p-2} d x\right)^{1 / p} \leqq A_{p}\|f\|_{p}
$$

for every such $f$.

THeOREM 3. Let $G$ be an infinite discrete abelian group containing $Z, Z\left(r^{\infty}\right)$ or $Z(r)^{\boldsymbol{N}_{0}}$ as a subgroup and $g$ an admissible function. Then $\left.g\right|_{(0,1)}=\left.\hat{f}^{*}\right|_{(0,1)}$ for some $f \in L^{p}(G)$ if and only if $\int_{0}^{1} g(x)^{p} x^{p-2} d x<$ $\infty$. Moreover there exists $A_{p}$ that depends only $p$ such that

$$
\left(\int_{0}^{1} g(x)^{p} x^{p-2} d x\right)^{1 / p} \leqq A_{p}\|f\|_{p}
$$

for every such $f$.

Theorems 1 and 2 give us a complete solution for all nondiscrete LCA groups. Theorem 3 holds for "almost all" discrete abelian groups, but I am not able to settle the case where $G$ contains $\Pi_{n=1}^{* \infty} Z\left(r_{n}\right)$ as a subgroup, with $r_{n} \rightarrow \infty$.

The forward implications " $\Rightarrow$ " of all three theorems and the existence of the constants $A_{p}$ are due to Hunt [5]; see Stein and Weiss [6], Chapter V, Corollary 3.16.

\section{A few lemmas.}

LEMMA 1. Let $G$ be a LCA group and $H$ an open subgroup of G. Let $H^{\perp}=\{\chi \in X: \chi=1$ on $H\}$. Then for each $f_{0} \in L^{p}(H)$, there exists $f \in L^{p}(G)$ such that $\hat{f}^{*}=\hat{f}_{0}^{*} m$ a.e. (where we use suitable Haar measures on $X$ and $X / H^{\perp}$ for the definitions of $\hat{f}^{*}$ and $\hat{f}_{0}^{*}$ ).

Proof. Let $f_{0} \in L^{p}(H)$ and define $f(x)=f_{0}(x)$ if $x \in H$ and $f(x)=0$ otherwise. Since $H$ is open, $f$ is still $\lambda$-measurable in $G$ 
and $f \in L^{p}(G)$. Choose Haar measure $\lambda_{H}$ on $H$ to be the restriction of $\lambda$ to $H$. Choose $\theta_{H^{\perp}}$ to be the normalized Haar measure on $H^{\perp}$, and $\theta_{X}$ to be an arbitrary Haar measure on $X$. Then a Haar measure $\theta_{1}$ on $X / H^{\perp}$ exists so that Weil's theorem applies [3; Vol. II, 28.54]. $\hat{f}$ is clearly constant on each coset of $H^{\perp}$. That is, $\hat{f}(\chi)=\hat{f}_{0}\left(\chi H^{\perp}\right)$ for all $\chi \in X$. A calculation, using Weil's theorem shows that $\hat{f}^{*}=$ $\hat{f}_{0}^{*} m$ a.e.

For the rest of this paper, we let $g$ be a fixed admissible function on $(0, \infty), 1<p<2$ and $\int_{0}^{\infty} g(x)^{p} x^{p-2} d x$ is finite.

LEMMA 2. (i) $\int_{0}^{1} g(c t) d m(t)<\infty$ for all $c>0$.

(ii) $0 \leqq \int_{0}^{\infty} g(c t) \sin x t d m(t) \leqq \int_{0}^{\pi / x} g(c t) \sin x t d m(t)<\infty$ for all $x>0, c>0$.

Proof. (i) Since

$$
\begin{aligned}
\int_{0}^{1} g(c t)^{p} d m(t) & \leqq \int_{0}^{1} g(c t)^{p} t^{p-2} d m(t) \leqq \int_{0}^{\infty} g(c t)^{p} t^{p-2} d m(t) \\
& =\frac{1}{c^{p-1}} \int_{0}^{\infty} g(t)^{p} t^{p-2} d m(t)<\infty,
\end{aligned}
$$

we see that $\int_{0}^{1} g(c t)^{p} d m(t)$ is finite and hence $\int_{0}^{1} g(c t) d m(t)$ is finite.

(ii) For $k=1,2, \cdots$, let

$$
\nu_{k}=(-1)^{k+1} \int_{(k-1) \pi / x}^{k \pi / x} g(c t) \sin x t d m(t) \text {. }
$$

It is clear that $\nu_{1} \geqq \nu_{2} \geqq \nu_{3} \geqq \cdots \geqq 0$ and $\nu_{k} \rightarrow 0$.

It follows that

$$
\int_{0}^{\infty} g(c t) \sin x t d t=\sum_{k=1}^{\infty}(-1)^{k+1} \nu_{k}
$$

and hence

$$
0 \leqq \int_{0}^{\infty} g(c t) \sin x t d t \leqq \nu_{1}=\int_{0}^{\pi / x} g(c t) \sin x t d m(t)<\infty .
$$

This completes the proof of Lemma 2 .

Define $G_{c}(x)=\int_{0}^{|x|} g(c t) d m(t)$ for $x \in R$. This is well-defined because $\int_{0}^{1} g(c t) d m(t)<\infty$ by (i) of Lemma 2 and $g$ is bounded in between 1 and $|x|$.

Lemma 3. (i) $G_{c}(x)=o\left(x^{1 / p}\right)$ as $x \rightarrow 0$ and as $x \rightarrow \infty$. 
(ii) $\int_{0}^{\infty} G_{c}(x)^{p} x^{-2} d m(x)<\infty$ for all $c>0$.

Proof. See [7], Vol. I, Ch. I, §9.16.

Lemma 4. There exists $f \in L^{p}(R)$ such that $\hat{f}^{*}=g m$ a.e.

Proof. Define, for $x \in R$

$$
\varphi(x)=\int_{0}^{\infty} g(2 t) \sin x t d m(t) .
$$

Then, by part (ii) of Lemma $20 \leqq \varphi(x) \leqq G_{2}(\pi / x)$, for $x>0$, because $0 \leqq \varphi(x) \leqq \int_{0}^{\pi / x} g(2 t) \sin x t d m(t) \leqq \int_{0}^{\pi / x} g(2 t) d m(t)=G_{2}(\pi / x)$. Since $G_{2}$ is an even function, we have that $|\varphi(x)| \leqq G_{2}(\pi / x)$ for all $x \in R \backslash\{0\}$. Part (ii) of Lemma 3 says that $G_{2}(\pi / x) \in L^{p}(R)$. If follows then that $\varphi \in L^{p}(R)$. Define, for $n \in N$,

$$
\varphi_{n}(x)=\int_{0}^{n} g(2 t) \sin x t d m(t) \quad(x \in R) .
$$

Let $x>0$. For each $n$, choose $m \in N$ such that $|2 m \pi / x-n| \leqq \pi / x$. Then

$$
\begin{aligned}
\left|\varphi_{n}(x)\right| & \leqq \int_{0}^{2 m \pi / x} g(2 t) \sin x t d m(t)+\left|\int_{2 m \pi / x}^{n} g(2 t) \sin x t d m(t)\right| \\
& \leqq \int_{0}^{\infty} g(2 t) \sin x t d m(t)+g\left(\frac{2(2 m-1) \pi}{x}\right)\left|\frac{2 m \pi}{x}-n\right| \\
& \leqq \varphi(x)+g\left(\frac{2 \pi}{x}\right) \frac{\pi}{x} \leqq \varphi(x)+\int_{0}^{\pi / x} g(2 t) d m(t) \\
& =\varphi(x)+G_{2}\left(\frac{\pi}{x}\right) .
\end{aligned}
$$

This shows that $\left|\varphi_{n}(x)\right| \leqq|\varphi(x)|+\left|G_{2}(\pi / x)\right|$ for all $x \in R \mid\{0\}$. Since $\varphi_{n}(x) \rightarrow \varphi(x)$ pointwise and $\varphi(x), G_{2}(\pi / x) \in L^{p}(R)$, we must have $\| \varphi_{n}$ $\varphi \|_{p} \rightarrow 0$ be the dominated convergence theorem. So we can obtain $\varphi$ by approximating $\varphi_{n}$. Let us compute $\varphi_{n}$ :

$$
\begin{aligned}
2 i \varphi_{n}(x)= & 2 i \int_{0}^{n} g(2 t) \sin x t d m(t)=\int_{0}^{n} g(2 t)\left(e^{-i x t}-e^{i x t}\right) d m(t) \\
= & \int_{R} g(-2 t) I_{[-n, 0]}(t) e^{-i x t} d m(t) \\
& -\int_{R} g(2 t) I_{[0, n]}(t) e^{-i x t} d m(t) .
\end{aligned}
$$

Recall that the Haar measure $m$ on $R$ is chosen so that the inversion theorem holds. We know that $g(2 t) I_{[0, n]}(t)$ and $g(-2 t) I_{[-n, 0]}(t) \in$ 
$L^{1}(R)$ and $\varphi_{n} \in L^{p}(R)$. Hence, by [3; Vol. II, 31.44 (b)], we have

$$
2 i \varphi(x)=\left\{\begin{array}{lll}
-g(2 x) & \text { if } & x \geqq 0 \\
g(-2 x) & \text { if } & x<0
\end{array}\right. \text { a.e. }
$$

Now define $f=2 i \varphi$ so that $|\hat{f}(x)|=g(|2 x|) m$ a.e. It is then easy to check that $\hat{f}^{*}=g m$ a.e., which is what we needed to prove.

Lemma 5. For each $n \in N$, there exists $f \in L^{p}\left(R^{n}\right)$ such that $\hat{f}^{*}=g m$ a.e.

Proof. By Lemma 4, we may assume that $n>1$. Define, for $k \in N$,

$$
\begin{aligned}
& \varphi(x)=\int_{0}^{\infty} g\left(2^{n} t\right) \sin x t d m(t) \\
& \varphi_{k}(x)=\int_{0}^{k} g\left(2^{n} t\right) \sin x t d m(t) \\
& f\left(x_{1}, \cdots, x_{n}\right)=2^{n} i \varphi\left(x_{i}\right) \frac{\sin x_{2}}{x_{2}} \cdots \frac{\sin x_{n}}{x_{n}} \\
& f_{k}\left(x_{1}, \cdots, x_{n}\right)=2^{n} i \varphi_{k}\left(x_{1}\right) \frac{\sin x_{2}}{x_{2}} \cdots \frac{\sin x_{n}}{x_{n}}
\end{aligned}
$$

Let $m_{n}=m \times m \times \cdots \times m$ on $R^{n}, x=\left(x_{1}, \cdots, x_{n}\right)$. Then

$$
\varphi(x), \varphi_{k}(x), \frac{\sin x}{x} \in L^{p}(R) \text {. }
$$

Therefore

$$
\begin{aligned}
\int_{R^{n}} \mid & f_{k}-\left.f\right|^{p} d m_{n} \\
& =2^{n^{p}} \int_{R^{n}}\left|\varphi_{k}\left(x_{1}\right)-\varphi\left(x_{1}\right)\right|^{p}\left|\frac{\sin x_{2}}{x_{2}}\right|^{p} \cdots\left|\frac{\sin x_{n}}{x_{n}}\right|^{p} d m_{n} \\
& =2^{n^{p}} \int_{R}\left|\varphi_{k}-\varphi\right|^{p} d m\left(\int_{R}\left|\frac{\sin x}{x}\right|^{p} d m\right)^{n-1} .
\end{aligned}
$$

As in the proof of Lemma 4 , we have $\left\|\varphi_{k}-\varphi\right\|_{p} \rightarrow 0$, and so $\| f_{k}$ $f \|_{p} \rightarrow 0$ in $L^{p}\left(R^{n}\right)$. Straight forward calculations show that

$$
\hat{f}_{k}\left(x_{1}, \cdots, x_{n}\right)=\left\{\begin{array}{l}
g\left(-2^{n} x_{1}\right) \text { if }-k \leqq x_{1}<0 \text { and } x_{j} \in[-1,1] \\
\quad \text { for } 2 \leqq j \leqq n \\
-g\left(2^{n} x_{1}\right) \text { if } 0 \leqq x_{1} \leqq k \text { and } x_{j} \in[-1,1] \\
\text { for } 2 \leqq j \leqq n \\
0 \quad \text { otherwise }
\end{array}\right.
$$


$m_{n}$ a.e. and hence

$$
\hat{f}\left(x_{1}, \cdots, x_{n}\right)=\left\{\begin{array}{l}
g\left(-2^{n} x_{1}\right) \text { if } x_{1}<0,\left|x_{j}\right| \leqq 1,2 \leqq j \leqq n \\
-g\left(2^{n} x_{1}\right) \text { if } x_{1}>0,\left|x_{j}\right| \leqq 1,2 \leqq j \leqq n \\
0 \text { otherwise }
\end{array}\right.
$$

$m_{n}$ a.e. It follows that

$$
\mathrm{m}_{n}\left\{x \in R^{n}:|\hat{f}(x)|>t\right\}=2^{n} m\left\{x_{1}>0: g\left(2^{n} x_{1}\right)>t\right\} .
$$

This in turn shows that for $x>0$

$$
\hat{f}^{*}(x)=\inf \left\{t>0: 2^{n} m\left\{x_{1}>0: g\left(2^{n} x_{1}\right)>t\right\} \leqq x\right\}=g(x)
$$

$m$ a.e., which completes the proof of Lemma 5 .

III. Proof for the nondiscrete case. Let $G$ be an infinite LCA group. To prove Theorem 1 and Theorem 2, Lemma 1 and the structure theorem [3, Vol. I, 24.30] shows that we may assume $G=$ $K \times R^{n}$, where $K$ is a compact abelian group.

Proof of Theorem 1. In this $n=0$, so that $G=K$. Then there exists $f_{0} \in L^{p}(K)$, by [4], such that $\left.\hat{f}_{0}^{*}\right|_{N}=\left.g\right|_{N}$.

Proof of Theorem 2. In this case $n>0$. By Lemma 5, there exists $f_{0} \in L^{p}\left(R^{n}\right)$ such that $\hat{f}_{0}^{*}=g m$ a.e. Define $f(x, y)=f_{0}(y)$ for $x \in K$ and $y \in R^{n}$. Let $m_{n}=m \times \cdots \times m$ be the Haar measure on $R^{n}, \lambda_{K}$ be the normalized Haar measure on $K$ and $\lambda_{K \times R^{n}}$ the Haar measure on $K \times R^{n}$ so that Weil's theorem holds. It follows that $f$ is in $L^{p}\left(K \times R^{n}\right)$ and $\|f\|_{p}=\left\|f_{0}\right\|_{p}$. Moreover, for $\chi_{1} \in \hat{K}, \chi_{2} \in R^{n}$, we have

$$
f\left(\chi_{1} \chi_{2}\right)=\left\{\begin{array}{l}
f_{0}\left(\chi_{2}\right) \text { if } \chi_{1}=1 \\
0 \text { otherwise }
\end{array}\right.
$$

Choose $\theta_{\hat{K} \times R} n, \theta_{\hat{K}}$ and $\theta_{R^{n}}$ the Haar measures on $\hat{K} \times R^{n}, \hat{K}$ and $R^{n}$ respectively, so that Planchevel's theorem holds. Then Weil's theorem holds for these measures by $[3,31.46(\mathrm{c})]$. Clearly $\theta_{\hat{K}}$ is the discrete measure on $\hat{K}$. Then for $t>0$

$$
\begin{aligned}
& \left(\theta_{\hat{K} \times R^{n}}\right)_{\hat{f}}(t)=\int_{\hat{K} \times R^{n}} I_{\{\chi:|\hat{f}(x)|>t\}} d \theta_{\hat{K} \times R^{n}} \\
& =\int_{R^{n}} \int_{\hat{K}} I_{\{x:|\hat{f}(x)|>t\}} d \theta_{\hat{K}} d \theta_{R^{n}} \\
& =\int_{R^{n}} I_{\left\{x:\left|\hat{f}_{0}(x)\right|>t\right\}} d \theta_{R^{n}}=\left(\theta_{R^{n}}\right)_{\hat{f}_{0}}(t),
\end{aligned}
$$

and it follows that for $x>0$, 


$$
\begin{aligned}
\hat{f}^{*}(x) & =\inf \left\{t>0:\left(\theta_{\hat{K} \times R^{n}}\right)_{\hat{f}}(t) \leqq x\right\}=\inf \left\{t>0:\left(\theta_{R^{n}}\right)_{\hat{f}_{0}}(t) \leqq x\right\} \\
& =\hat{f}_{0}^{*}(x)=g(x) m \text { a.e. }
\end{aligned}
$$

Note that Theorem 1 is essentially the theorem in [4].

IV. Proof of Theorem 3. For each $n=1,2, \cdots$, let $r_{n}$ be an integer $\geqq 2$. Denote by $\theta$ the normalized Haar measure on $X=$ $\prod_{n=1}^{\infty} Z\left(r_{n}\right)$ and $\lambda$ the usual restriction of Lebsque measure to $[0,1]$. Define a function $\varphi: X \rightarrow[0,1]$ via

$$
\varphi(\varepsilon)=\sum_{n=1}^{\infty} \frac{\varepsilon_{n}}{p_{1} p_{2} \cdots p_{n}} \quad \varepsilon=\left(\varepsilon_{1}, \cdots, \cdots\right) \in X .
$$

Then $g$ is measure preserving; in fact, the following is well known.

Lemma 6. $E$ is measurable in $X$ if and only if $\varphi(E)$ is measurable in $[0,1]$, and $\theta(E)=\lambda(\varphi(E))$. $\varphi$ is an onto map and $\varphi$ is one-to-one on $X$ except for a countable set. Moreover,

$$
\int_{x} h \circ \varphi d \theta=\int_{0}^{1} h d \lambda
$$

for all bounded $\lambda$ measurable functions $h$ on $[0,1]$.

LEMMA 7. Theorem 3 is true if $G \supset Z$.

Proof. By Lemma 1, we may assume $G=Z$. Define

$$
a_{0}(n)=\frac{1}{2 \pi} \int_{0}^{2 \pi} g(t) \sin n t d t \quad \text { for } n \in Z \text {. }
$$

The values of the integrals involved are finite, by (i) of Lemma 2. Also $a_{0} \in l^{p}(Z)$ because

$$
\begin{gathered}
(2 \pi)^{p} \sum_{n \in Z}\left|a_{0}(n)\right|^{p}=\sum_{n \in Z}\left|\int_{0}^{2 \pi} g(t) \sin n t d t\right|^{p} \leqq \sum_{\substack{n \in Z \\
n \neq 0}}\left|\int_{0}^{\pi / n} g(t) d t\right|^{p} \\
=\sum_{\substack{n \in Z \\
n \neq 0}} G_{1}\left(\frac{\pi}{n}\right)^{p} \leqq \int_{R} G_{1}^{p}\left(\frac{\pi}{x}\right) d x=\pi \int_{R} G_{1}^{p}(y) y^{-2} d y .
\end{gathered}
$$

The last integral is finite by (ii) of Lemma 3. Similarly, if we define

$$
b_{0}(n)=\frac{1}{2 \pi} \int_{0}^{2 \pi} g(t) \cos n t d t \quad \text { for } n \in Z
$$

then $b_{0} \in l^{p}(Z)$. So if we set $c(n)=b_{0}(n)-i a_{0}(n)=1 / 2 \pi \int_{0}^{2 \pi} g(t) e^{-i n t} d t$ for $n \in Z$, then $c \in l^{p}(Z)$ and $\hat{c}(t)=g(t)$ a.e. [3, 31.44, (b)]. Since $g$ is nonincreasing in $[0,2 \pi]$, we then have $\hat{c}^{*}=g \theta$ a.e. 
LEMMA 8. Theorem 3 is true is $G \supset \Pi^{*} Z(r)$, where $r \in N, r \geqq 2$.

Proof. We may assume that $G=\Pi^{*} Z(r)$, by Lemma 1 .

Let $X=Z(r)^{\aleph_{0}}$, the character group of $G$. Define $\varphi(\varepsilon)=\sum_{n=1}^{\infty} \varepsilon_{n} / r_{n}$ for $\varepsilon=\left(\varepsilon_{n}\right) \in X$, and note that Lemma 6 applies to $\varphi$. For a real number $t$, denote $[t]$ by the greatest integer which is not greater than $t$. For $m \in N$, define

$$
\chi_{m}(t)=e^{i 2 \pi\left[r^{m} t\right] / r}
$$

for $t \in[0,1]$. Then $\chi_{m} \circ \varphi(\varepsilon)=e^{i(2 \pi / r) \varepsilon_{m}}$ where $\varepsilon \in X$ and $\varepsilon_{m}$ is the $m$ th component of $\varepsilon$. It follows that $G$ is isomorphic to the group of finite products of elements in $\left\{\chi_{m} \circ \varphi\right\}_{m=1}^{\infty}$. In this proof we write $I_{m, \nu}$ for the characteristic function of the interval $\left[v / r^{m},(v+1) / r^{m}\right]$

$$
\chi_{m}(t)=\sum_{u=1}^{r-1}\left(\sum_{j=0}^{r-1} w^{j} I_{m,(u-1) r+j}(t)\right)
$$

for $\theta$ a.e. $t$, where $w=e^{i(2 \pi / r)}$. And hence

$$
\chi_{m_{1}}^{l_{1}}(t) \cdots \chi_{m_{k}}^{l_{k}}(t)=\sum_{u=1}^{r_{m_{1}-1}} a_{u}\left(\sum_{j=0}^{r-1} w^{l_{1} j} I m_{1},(u-1) r+j(t)\right)
$$

where $a_{u}^{r}=1$ for all $u=1, \cdots, r^{m_{1}-1} ; m_{1}>m_{2}>\cdots>m_{k}$ and $0 \leqq l_{1}$, $l_{2}, \cdots, l_{k} \leqq r-1, l_{1}>0$.

Define a function $f$ on $G$ via

$$
f\left(\chi_{m_{1}}^{l_{1}} \circ \varphi, \cdots, \chi_{m_{k}}^{l_{k}} \circ \varphi\right)=\int_{X} g \circ \varphi(\varepsilon) \chi_{m_{1}}^{l_{1}} \circ \varphi(\varepsilon) \cdots \chi_{m_{k}}^{l_{k}} \circ \varphi(\varepsilon) d \varepsilon .
$$

Define, for $u=1,2, \cdots, r^{m_{1}-1}$ and $j=0, \cdots, r-1$,

$$
k_{(u-1) r+j}=\int I_{m_{1},(n-1) r+j}(t) g(t) d t, b_{(u-1) r+j}=a_{u} w^{j l_{1}} .
$$

Then $\left\{k_{0}, k_{1}, \cdots, k_{r^{m_{1-1}}}\right\}$ is a positive nonincreasing sequence, and

$$
\left|\sum_{l=0}^{s} b_{l}\right| \leqq r \text { for all } s=0,1,2, \cdots, r^{m_{1}}-1
$$

In fact,

$$
\sum_{j=0}^{r-1} b_{(u-1) r+j}=\sum_{j=0}^{r-1} a_{u} w^{j l_{1}}=a_{u} \sum_{j=0}^{r-1} w^{j l_{1}}=0 .
$$

It follows that

$$
\mid f\left(\chi_{m_{1}}^{l_{1}} \circ \varphi, \cdots, \chi_{m_{k}}^{\left.l_{k} \circ \varphi\right)}|=| \int_{0}^{1} g(t) \chi_{m_{1}}^{l_{1}}(t), \cdots, \chi_{m_{k}}^{l_{k}}(t) d t \mid\right.
$$




$$
\begin{aligned}
& =\sum_{u=1}^{r^{m_{1}-1}}\left(\sum_{j=0}^{r-1} a_{u} w^{j l_{1}} \int I_{m_{1},(u-1) r+j}(t) g(t) d t\right)=\left|\sum_{l=0}^{r^{m_{1}-1}} b_{l} k_{l}\right| \\
& \leqq k_{0} \max _{0 \leqq s \leqq r^{m_{1}}}\left|\sum_{l=0}^{s} b_{l}\right| \leqq k_{0} r=r \int_{0}^{1 / r^{m_{1}}} g(t) d t=r G\left(\frac{1}{r^{m_{1}}}\right) .
\end{aligned}
$$

Writing $\Sigma^{\prime}$ for a sum over all $\left(m_{1}, \cdots, m_{k}, l_{1}, \cdots, l_{k}\right)$ satisfying $k \in$ $N, m_{1}>m_{2}>\cdots>m_{k} \geqq 0,0<l_{1} \leqq r-1,0 \leqq l_{j} \leqq r-1$ for $j=2$, $\cdots, k$, we obtain

$$
\begin{aligned}
\|f\|_{p}^{p} & =\Sigma^{\prime}\left|f\left(\chi_{m_{1}}^{l_{1}} \varphi, \cdots, \chi_{m_{k}}^{l_{k}} \varphi\right)\right|^{p} \leqq \Sigma^{\prime} r^{p} G^{p}\left(\frac{1}{r^{m_{1}}}\right) \\
& \leqq \sum_{m_{1}=0}^{\infty} r^{m_{1}} r^{p} G^{p}\left(\frac{1}{r^{m_{1}}}\right)=r^{p+1} \sum_{m_{1}=0}^{\infty} r^{m_{1}-1} G^{p}\left(\frac{1}{r^{m_{1}}}\right) \\
& \leqq r^{p+1} \sum_{m_{1}=0}^{\infty}\left(r^{m_{1}}-r^{m_{1}-1}\right) G^{p}\left(\frac{1}{r^{m_{1}}}\right) \leqq r^{p+1} \int_{0}^{\infty} G^{p}\left(\frac{1}{x}\right) d x<\infty .
\end{aligned}
$$

So $f \in L^{p}(G)$ and hence $\hat{f}=g \circ \varphi$. It follows that $\hat{f}^{*}=g I_{[0,1]} m$ a.e.

Lemma 9. Theorem 3 is true if $G$ contains $Z\left(r^{\infty}\right),(r \geqq 2)$.

Proof. We may assume that $G=Z\left(r^{\infty}\right)$ by Lemma 1. Let $\Delta_{r}$ be the group of $r$-adic integers; then $Z\left(r^{\infty}\right)$ is a discrete group with $Z\left(r^{\infty}\right)^{\wedge}=\Delta_{r}$. Define

$$
\varphi(\varepsilon)=\sum_{n=1}^{\infty} \frac{\varepsilon_{n}}{r^{n}} \varepsilon=\left(\varepsilon_{n}\right) \in \Delta_{r} .
$$

As in Lemma $6, \varphi$ is a measure preserving map from $\Delta_{r}$ onto $[0,1]$, and

$$
\int_{\Delta_{r}} h \circ \varphi d^{\theta}=\int_{0}^{1} h d t
$$

for all bounded measurable functions $h$ on $[0,1]$, where $\theta$ is the normalized Haar measures on $\Delta_{r}$. We write $I_{m, s_{1}, \ldots, s_{m}}$ for the characteristic function of the interval

$$
\left[\frac{r^{m-1} s_{1}+r^{m-2} s_{2}+\cdots+s_{m}}{r^{m}}, \frac{r^{m-1} s_{1}+r^{m-2} s_{2}+\cdots+s_{m}+1}{r^{m}}\right] .
$$

For $m \in N$, define

$$
\chi_{m}(t)=\sum_{s_{1} \cdots s_{m}=0}^{r-1} w_{m}^{s_{1}+r s_{2}+\cdots+r^{m-1} s_{m}} I_{m, s_{1}, \cdots, s_{m}}(t)
$$

where $w_{m}=e^{i\left(2 \pi / r^{m}\right)}$. Then $\chi_{m} \circ \varphi(\varepsilon)=w_{m}^{\varepsilon_{1}+r \varepsilon_{2}+\cdots+r^{m-1}} \varepsilon_{m} \theta$ a.e. where $(\varepsilon) \epsilon$ $\Delta_{r}$ and $\varepsilon_{1}, \cdots, \varepsilon_{m}$ are the first $m$ coordinates of $(\varepsilon)$. It follows that $G$ is isomorphic to the group generated by $\left\{\chi_{m} \circ \varphi\right\}_{m=1}^{\infty}$. Define for 
$m, l \in N$ and $(l, r)=1$

$$
f\left(\chi_{m}^{l}\right)=\int_{\Delta_{r}} g \circ \phi(\varepsilon) \chi_{m}^{l} \circ \phi(\varepsilon) d \theta
$$

Then $f$ is a function on $G$, and by (2) and (3),

$$
\begin{aligned}
f\left(\chi_{m}^{l}\right) & =\int_{0}^{1} g(t) \chi_{m}^{l}(t) d t \\
& =\sum_{s_{1}, \cdots, s_{m}=0}^{r-1}\left(w_{m}^{l}\right)^{s_{1}+r_{s_{2}}+\cdots+r^{m-1} s_{m}} \int I_{m, s_{1}, \cdots, s_{m}}(t) g(t) d t .
\end{aligned}
$$

Let $k_{r^{m-1} s_{1}+\cdots+s_{m}}=\int I_{m, s_{1}, \cdots, s_{m}}(t) g(t) d t$. Then $\left\{k_{0}, k_{1}, \cdots, k_{r^{m}-1}\right\}$ is a positive, nonincreasing sequence. Let $b_{r^{m-1} s_{1}+\cdots+s_{m}}=\left(w_{w}^{l}\right)^{s_{1}+r s_{2}+\cdots+r^{m-1} s_{m}}$. For any $0 \leqq s \leqq r^{m}-1$, we write $s=r^{m-1} s_{1}+\cdots+s_{m}$ with $0 \leqq s_{1}, \cdots$, $s_{m}<r$. Then

$$
\begin{aligned}
\sum_{n=0}^{s} b_{n} & =\sum_{n=1}^{r m-1 s_{1}+\cdots+s_{m}} b_{n} \\
& =\left(\sum_{u=1}^{r m-2_{s_{1}}+\cdots \cdot \varphi_{m-1}} \sum_{h=0}^{r-1} b_{(u-1) r+h}\right)+\left(\sum_{j=0}^{s_{m}} b_{r^{m-1} s_{1}+\cdots+r s_{m-1}+j}\right)
\end{aligned}
$$

For each $u=1, \cdots, r^{m-2} s_{1}+\cdots+s_{m-1}$. Choose $0 \leqq u_{1}, \cdots, u_{m-1}<r$ such that $(u-1) r=r^{m-1} u_{1}+\cdots+r u_{m-1}$, and hence

$$
\begin{aligned}
& \sum_{h=0}^{r-1} b_{(u-1) r+h}=\sum_{h=0}^{r-1} b_{r^{m-1} u_{1}+\cdots+r u_{m-1}+h} \\
& =\sum_{h=0}^{r-1}\left(w_{m}^{l}\right)^{u_{1}+r u_{2}+\cdots+r^{m-2} u_{m-1}+r m-1 h} \\
& =\left(w_{m}^{l}\right)^{u_{1}+r u_{2}+\cdots+r^{m-2} u_{m-1}} \sum_{h=0}^{r-1}\left(w_{m}^{l}\right) r^{m-1 h} \\
& =\left(w_{m}^{l}\right)^{u_{1}+r u_{2}+\cdots+r^{m-2} u_{m-1}} \sum_{h=0}^{r-1}\left(e^{i(2 \pi l) / r}\right)^{h}=0 .
\end{aligned}
$$

The last equality holds because $(l, r)=1$. This shows that

$$
\left|\sum_{n=0}^{s} b_{n}\right|=\left|\sum_{j=0}^{s_{m}} b_{r^{m-1} s_{1}+\cdots+r s_{m-1}+j}\right| \leqq s_{m}+1 \leqq r
$$

and hence

$$
\begin{aligned}
\left|f\left(\chi_{m}^{l}\right)\right| & =\left|\sum_{n=0}^{r m-1} b_{n} k_{n}\right| \leqq k_{0} \max _{0 \leqq s \leqq r^{m}-1}\left|\sum_{n=0}^{s} b_{n}\right| \leqq r k_{0} \\
& =r \int_{0}^{1 / r m} g(t) d t=r G_{1}\left(\frac{1}{r^{m}}\right)
\end{aligned}
$$

for all $m, l \in N$ and $(l, r)=1$. Denote by $\Sigma^{\prime}$ the sum over $(m, l) \in N$, $(l, r)=1$ and $0 \leqq l<r^{m}$. Then we have 


$$
\|f\|_{p}^{p}=\Sigma^{\prime}\left|f\left(\chi_{m}^{l}\right)\right|^{p} \leqq \Sigma^{\prime} r^{p} G_{1}^{p}\left(\frac{1}{r^{m}}\right) \leqq \sum_{m=0}^{\infty} r^{m} r^{p} G_{1}^{p}\left(\frac{1}{r^{m}}\right) \text {. }
$$

As in Lemma 8, we conclude that $f \in L^{p}(G)$ and $\hat{f}^{*}=g I_{[0,1]} m$ a.e.

Patching Lemmas 7, 8 and 9 together gives the proof of Theorem 3.

I would like to extend my sincere thanks here to Professor K. A. Ross for his helpful suggestions.

The remaining open question is whether Theorem 3 holds if $G=$ $\Pi_{n=1}^{\infty} * Z\left(r_{n}\right)$ where $r_{n} \in N, r_{n} \geqq 2$ for all $n$ and $\lim _{n \rightarrow \infty} r_{n}=\infty$.

\section{REFERENCES}

1. G. H. Hardy and J. E. Littlewood, some new properties of Fourier constants, Math. Annalen, 97 (1926), 159-209.

2. - some new propeties of Fourier constants, J. London Math. Soc., 6 (1931), 3-9.

3. E. Hewitt and K. A. Ross, Abstract Harmonic Analysis, 2 vols. (Springer-Verlag, Inc. Berlin-Heidelberg-New York 1963, 1970).

4. - Rearrangement of $L^{r}$ Fourier Series on compact abeliangroups, Proc. London Math. Soc., (3) 29 (1974), 317-330.

5. R. A. Hunt, On $L(p, g)$ spaces, $L^{\prime}$ Enseignement Math., 12 (1966), 249-275.

6. E. M. Stein and G. Weiss, Introduction to Fourier Analysis on Euclidean Spaces. (Princeton, New Jersey, Princeton University Press, 1971).

7. A. Zygmund, Trigonometric Series, 2 nd edition, 2 vols. (Cambridge Press 1959, reprinted 1968).

Received February 20, 1975.

UNVERSITY OF OREGON 



\section{PACIFIC JOURNAL OF MATHEMATICS}

\section{EDITORS}

RICHARD ARENS (Managing Editor)

University of California

Los Angeles, California 90024

\section{J. DugundJI}

Department of Mathematics University of Southern California Los Angeles, California 90007

D. Gilbarg and J. Milgram

Stanford University

Stanford, California 94305
University of Washington Seattle, Washington 98105

\section{ASSOCIATE EDITORS}
E. F. BECKENBACH
B. H. NeumanN
F. WolF
K. YoShIDA

\section{SUPPORTING INSTITUTIONS}

\author{
UNIVERSITY OF SOUTHERN CALIFORNIA \\ STANFORD UNIVERSITY \\ UNIVERSITY OF TOKYO \\ UNIVERSITY OF UTAH \\ WASHINGTON STATE UNIVERSITY \\ UNIVERSITY OF WASHINGTON \\ $\stackrel{*}{*} \stackrel{*}{*} \stackrel{*}{ }{ }^{*}$ AMERICAN MATHEMATICAL SOCIETY
}

The Supporting Institutions listed above contribute to the cost of publication of this Journal, but they are not owners or publishers and have no responsibility for its content or policies.

Mathematical papers intended for publication in the Pacific Journal of Mathematics should be in typed form or offset-reproduced, (not dittoed), double spaced with large margins. Underline Greek letters in red, German in green, and script in blue. The first paragraph or two must be capable of being used separately as a synopsis of the entire paper. Items of the bibliography should not be cited there unless absolutely necessary, in which case they must be identified by author and Journal, rather than by item number. Manuscripts, in triplicate, may be sent to any one of the editors. Please classify according to the scheme of Math. Reviews, Index to Vol. 39. All other communications should be addressed to the managing editor, or Elaine Barth, University of California, Los Angeles, California, 90024.

The Pacific Journal of Mathematics expects the author's institution to pay page charges, and reserves the right to delay publication for nonpayment of charges in case of financial emergency.

100 reprints are provided free for each article, only if page charges have been substantially paid. Additional copies may be obtained at cost in multiples of 50 .

The Pacific Journal of Mathematics is issued monthly as of January 1966. Regular subscription rate: $\$ 72.00$ a year (6 Vols., 12 issues). Special rate: $\$ 36.00$ a year to individual members of supporting institutions.

Subscriptions, orders for back numbers, and changes of address should be sent to Pacific Journal of Mathematics, 103 Highland Boulevard, Berkeley, California, 94708.

\section{PUBLISHED BY PACIFIC JOURNAL OF MATHEMATICS, A NON-PROFIT CORPORATION}

Printed at Kokusai Bunken Insatsusha (International Academic Printing Co., Ltd.), 270, 3-chome Totsuka-cho, Shinjuku-ku, Tokyo 160, Japan.

\section{Copyright (C) 1975 by Pacific Journal of Mathematics} Manufactured and first issued in Japan 


\section{Pacific Journal of Mathematics}

\section{Vol. 57, No. $2 \quad$ February, 1975}

Norman Larrabee Alling, On Cauchy's theorem for real algebraic curves with boundary .......

Daniel D. Anderson, A remark on the lattice of ideals of a Prüfer domain ..................

Dennis Neal Barr and Peter D. Miletta, A necessary and sufficient condition for uniqueness of

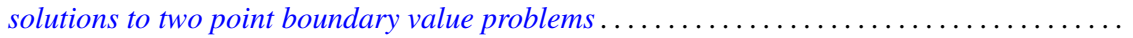

Ladislav Beran, On solvability of generalized orthomodular lattices . . . . . . . . . . ........

L. Carlitz, A three-term relation for some sums related to Dedekind sums . . . . . . . . . .....

Arthur Herbert Copeland, Jr. and Albert Oscar Shar, Images and pre-images of localization

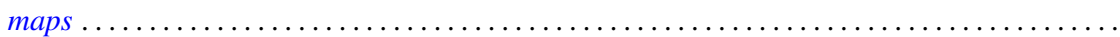

G. G. Dandapat, John L. Hunsucker and Carl Pomerance, Some new results on odd perfect

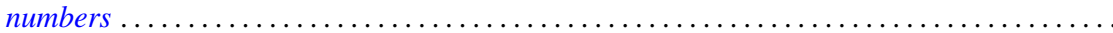

M. Edelstein and L. Keener, Characterizations of infinite-dimensional and nonreflexive

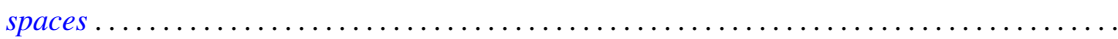

Francis James Flanigan, On Levi factors of derivation algebras and the radical embedding

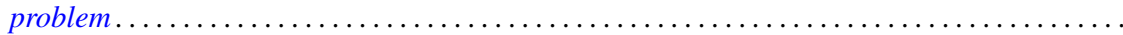

Harvey Friedman, Provable equality in primitive recursive arithmetic with and without

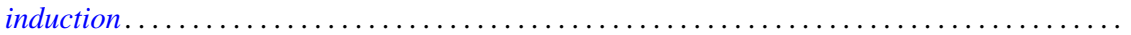

Joseph Braucher Fugate and Lee K. Mohler, The fixed point property for tree-like continua with

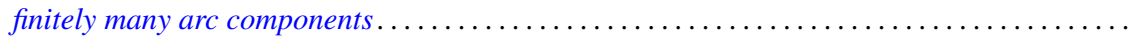

John Norman Ginsburg and Victor Harold Saks, Some applications of ultrafilters in

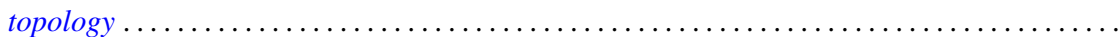

Arjun K. Gupta, Generalisation of a "square" functional equation .....................

Thomas Lee Hayden and Frank Jones Massey, Nonlinear holomorphic semigroups ..........

V. Kannan and Thekkedath Thrivikraman, Lattices of Hausdorff compactifications of a locally

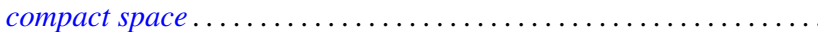

J. E. Kerlin and Wilfred Dennis Pepe, Norm decreasing homomorphisms between group

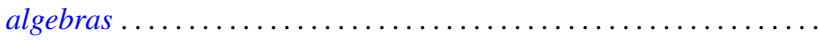

Young K. Kwon, Behavior of $\Phi$-bounded harmonic functions at the Wiener boundary ...

Richard Arthur Levaro, Projective quasi-coherent sheaves of modules .

Chung Lin, Rearranging Fourier transforms on groups...........................

David Lowell Lovelady, An asymptotic analysis of an odd order linear differential equation . . 4475

Jerry Malzan, On groups with a single involution .......................... 481

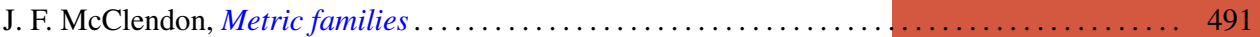

Carl Pomerance, On multiply perfect numbers with a special property .

Mohan S. Putcha and Adil Mohamed Yaqub, Polynomial constraints for finiteness of semisimple rings. .

Calvin R. Putnam, Hyponormal contractions and strong power convergence . . . . . . . . . 531

Douglas Conner Ravenel, Multiplicative operations in $\mathrm{BP} * \mathrm{BP} \ldots \ldots \ldots \ldots \ldots \ldots \ldots \ldots \ldots .539$

Judith Roitman, Attaining the spread at cardinals which are not strong limits . . . . . . . . . 545

Kazuyuki Saitô, Groups of *-automorphisms and invariant maps of von Neumann algebras . . . 553

Brian Kirkwood Schmidt, Homotopy invariance of contravariant functors acting on smooth

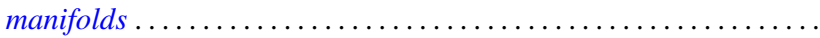

Kenneth Barry Stolarsky, The sum of the distances to $N$ points on a sphere.

Mark Lawrence Teply, Semiprime rings with the singular splitting property.

J. Pelham Thomas, Maximal connected Hausdorff spaces..............

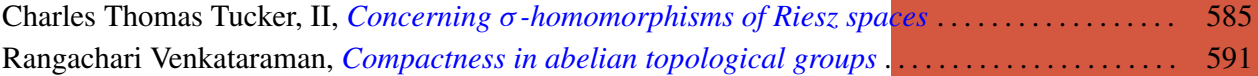

William Charles Waterhouse, Basically bounded functors and flat sheaves . . . . . . . . . . . 597

David Westreich, Bifurcation of operator equations with unbounded linearized part ......... 611

William Robin Zame, Extendibility, boundedness and sequential convergence in spaces of 